\title{
Implementação e avaliação de um curso blended learning de Bioquímica para estudantes de Odontologia
}

\author{
Paulo Henrique Tomazinho*; Thais Mariana Neves Chaves**; Flávia Sens Fagundes***; Marilisa Caneiro \\ Leão Gabardo***; Eduardo Pizzatto***, Carla Castiglia Gonzaga***; João Armando Brancher*** \\ * Doutorando em Odontologia, Escola de Ciências da Saúde, \\ Universidade Positivo \\ ** Mestrando, Mestrado Profissional em Odontologia Clínica, \\ Escola de Ciências da Saúde, Universidade Positivo \\ *** Docente, Mestrado Profissional em Odontologia Clínica e \\ Doutorado em Odontologia Escola de Ciências da Saúde, \\ Universidade Positivo
}

Recebido em 15/01/2018. Aprovado em 09/12/2018.

\begin{abstract}
RESUMO
O objetivo deste estudo foi avaliar o desempenho de estudantes do curso de Odontologia na disciplina de Bioquímica por meio do uso da metodologia Blended Learning. Para isso formaram-se dois grupos: um composto por estudantes que cursaram a disciplina de Bioquímica no formato tradicional no ano de 2015, denominado grupo em classe (EC); e outro com aqueles que cursaram a mesma disciplina no formato Blended Learning no ano de 2016, denominado grupo BL. Os objetivos de aprendizagem, o conteúdo apresentado e as avaliações foram os mesmos para ambos. O desempenho dos estudantes foi mensurado por meio de avaliações bimestrais presenciais e individuais com níveis de dificuldade semelhantes entre os grupos. Os resultados obtidos foram analisados utilizando o teste ANOVA a dois fatores, com nível de significância de 0,05. No ano de 2015 participaram da pesquisa 198 estudantes, 54,4\% do gênero feminino e 45,5\% do gênero masculino. No ano de 2016, 148 estudantes participaram da pesquisa, também com predomínio de estudantes do gênero feminino $(52,7 \%)$. Os estudantes do grupo BL obtiveram melhores resultados no $1^{\circ}, 2^{\circ}$ e $3^{\circ}$ bimestres $(\mathrm{p}<0,05)$. A média anual dos estudantes do grupo BL também foi melhor do que a média do grupo EC. Este estudo sugere que a metodologia Blended Learning propicie maior ganho de conhecimento aos estudantes quando comparada à metodologia de ensino centrada em aulas presenciais.

Descritores: Educação. Odontologia. Metodologias Ativas.
\end{abstract}




\section{INTRODUÇÃO}

O ensino da Odontologia no Brasil é regulamentado pelo Conselho Nacional de Educação (CNE), órgão que delibera a respeito das diretrizes que devem ser implementadas nos cursos de graduação. De acordo com o artigo $3^{\circ}$ desse documento, graduandos em Odontologia devem trabalhar em todos os níveis da saúde com acurado rigor técnico e científico. Além disso, devem ser capazes de compreender a realidade social e cultural do ambiente no qual trabalham, portanto, precisam desenvolver habilidades que incluem a tomada de decisões ${ }^{1}$. Neste sentido, a educação continuada do próprio profissional é indispensável.

No que diz respeito à educação continuada, $\mathrm{o}$ documento é claro quando menciona que o profissional deve aprender a aprender, além de ter responsabilidade e compromisso com sua própria aprendizagem. Neste ponto pergunta-se: os graduandos em Odontologia têm capacidade de aprender sem as aulas tradicionais que são ministradas na universidade? A resposta a esta questão requer uma análise aprofundada dos currículos dos cursos de Odontologia, os quais deveriam oferecer aos discentes e aos docentes a oportunidade de vivenciar novos métodos de ensino e aprendizagem. Houve um progresso neste sentido em alguns países onde a formatação curricular aumentou as habilidades de comunicação e a reflexão crítica do aluno ${ }^{2}$, mas aprender a aprender ainda é uma deficiência curricular.

Neste contexto, as metodologias ativas associadas às tecnologias digitais são ferramentas úteis tanto para professores quanto para estudantes, uma vez que permitem interação, colaboração e cooperação de diferentes maneiras ${ }^{3,4}$. Blended Learning (BL) é uma dessas ferramentas que associa atividades on-line e aulas convencionais permitindo a criação de um ambiente de aprendizagem motivador ${ }^{5}$. Esta metodologia facilita $o$ desenvolvimento de competências e permite evitar a sobrecarga de informações na sala de aula ${ }^{6}$ porque mistura dois ou mais métodos de ensino e aprendizagem ${ }^{7}$. Sua implementação em disciplinas tais como anatomia ${ }^{8}$ e fisiologia ${ }^{9}$ permitiu diferentes abordagens, que vão além das aulas tradicionais, aumentando a performance dos estudantes, sua motivação e satisfação, ao mesmo tempo que permitiu uma flexibilização da aprendizagem. Além disso, com a entrada da educação superior na era digital, a metodologia Blended Learning pode ser uma maneira eficiente para economizar recursos, tanto das universidades quanto dos estudantes, e otimizar o processo ensino aprendizado 9 .

A disciplina de Bioquímica abrange um campo de atuação interdisciplinar que fornece subsídios para vários cursos da área de ciências da saúde tais como química médica e biologia molecular, entre outros ${ }^{10}$. O objetivo básico desta ciência é mostrar como moléculas que constituem os organismos vivos interagem para manter a vida das células $^{11} \mathrm{e}$, por consequência, do próprio corpo humano, o que faz com que ela assuma um papel relevante para o entendimento de outras ciências básicas. Ensinar e aprender bioquímica não é uma tarefa fácil e as principais dificuldades encontradas pelos estudantes parecem estar relacionadas com a formatação das aulas teóricas ${ }^{10}$. Nesse sentido, metodologias ativas de aprendizagem, associadas às tecnologias digitais podem favorecer $\mathrm{o}$ entendimento da Bioquímica. Dessa forma, o objetivo desta pesquisa foi implementar e avaliar a eficácia de um curso Blended Learning de Bioquímica desenvolvido para estudantes de Odontologia, combinando atividades on-line $\mathrm{e}$ atividades presenciais.

\section{METODOLOGIA}

Este é um estudo coorte de dois anos, conduzido com dois grupos de estudantes de Odontologia, ambos da Universidade Positivo, em Curitiba, Paraná. O primeiro grupo cursou a disciplina de Bioquímica no formato tradicional durante o ano letivo 2015, grupo em classe (EC). O 
outro grupo cursou a mesma disciplina no formato Blended Learning durante o ano de 2016 (Grupo BL).

Para medir a eficiência das metodologias adotadas, foram definidos resultados de aprendizagem iguais para os dois grupos que foram organizados hierarquicamente do menor grau de complexidade para o maior grau de complexidade, de acordo com a Taxonomia de Bloom (Bloom et al, 1956) $)^{12}$.

\section{Atividades propostas para o grupo em classe (EC)}

Para o grupo EC, todo o conteúdo de Bioquímica foi ministrado por um único professor em aulas presenciais de duas horas semanais durante as quarenta semanas letivas. As aulas foram expositivas e os estudantes tinham acesso irrestrito ao conteúdo da disciplina através do portal universitário e da plataforma digital da universidade. Além disso, artigos, textos, hiperlinks, imagens, exercícios de revisão e outros materiais de apoio foram oferecidos nessa plataforma. Os conteúdos trabalhados foram assim distribuídos:

$1^{\circ}$ Bimestre: Mecanismos de homeostasia celular, pH e tampões, equilíbrio ácido básico e bioenergética. Proteínas e enzimas; cinética enzimática; $2^{\circ}$ Bimestre: Carboidratos e glicólise; fermentações; respiração celular; $3^{\circ}$ Bimestre: Metabolismo de carboidratos, glicogenólise, glicogênese e gliconeogênese, lipídeos e lipoproteínas, metabolismo de lipoproteínas; e $4^{\mathrm{o}}$ Bimestre: Metabolismo de lipídeos; metabolismo de aminoácidos; mecanismo de regulação hormonal e inter-relação metabólica.

Os estudantes foram estimulados a elucidar suas dúvidas presencialmente, no final de cada aula ou via e-mail. Foram realizadas atividades em classe e atividades extraclasse em cada bimestre que foram utilizadas para compor cada nota bimestral. Ao conjunto dessas atividades foi atribuído o valor de $50 \%$ da nota.

\section{Atividades propostas para os estudantes do grupo BL}

Entre os meses de setembro de 2015 e fevereiro de 2016 um grupo de professores da Universidade formatou todo o conteúdo de Bioquímica em um e-book com oito capítulos que foi utilizado pelos estudantes durante todo o ano de 2016. Para cada capítulo foram criados mapas conceituais, vídeo-aulas e bancos de atividades online compostos por questões objetivas, discursivas e questões indutoras. Estas últimas foram utilizadas em fóruns de discussão on-line. Os estudantes também dispunham de um professor tutor on-line que os ajudavam na resolução de dúvidas e garantiam o feedback imediato para as dúvidas dos estudantes.

Antes das aulas presenciais o estudante deveria ler o capítulo, entender o mapa conceitual, assistir a vídeo-aula e resolver atividades on-line, sempre acompanhado por um tutor. Nas aulas presenciais o conteúdo de cada capítulo era abordado por meio de metodologias ativas de aprendizagem tais como instrução por pares, atividades ativas, grupos colaborativos e estudos de caso. $\mathrm{O}$ percurso de aprendizagem proposto para o estudante na modalidade BL está representado na figura 1.

Dessa forma os estudantes precisavam realizar atividades e exercícios prévios específicos para participar das aulas presenciais, cada uma dessas atividades foi utilizada para compor a nota bimestral. Atividades on-line e atividades extraclasse somaram $50 \%$ da nota. A tabela 1 mostra como foram distribuídos os conceitos em cada atividade para o grupo BL. Todas as atividades on-line ficaram sob responsabilidade do professor tutor enquanto as atividades presenciais ficaram sob responsabilidade do professor presencial.

\section{Avaliações Presenciais}

Tanto no ano de 2015 quanto no ano de 2016 foram aplicadas avaliações presenciais 
individuais com níveis de dificuldade semelhantes, sem nenhum tipo de discriminação entre os grupos. As avaliações presenciais contribuíram com $50 \%$ da nota total dos estudantes, sendo assim, em cada avaliação bimestral o estudante poderia alcançar no máximo 5,0 pontos. O desempenho individual dos estudantes nestas avaliações foi utilizado para mensurar ganho de conhecimento de ambos os grupos.

\section{Análise estatística}

Os resultados obtidos foram analisados utilizando o teste ANOVA a dois fatores (Statistica 10.0, StatSoft, Tulsa, OK, EUA), com nível de significância de 0,05 .

\section{RESULTADOS}

As características dos dois grupos estudados estão descritas na tabela 2. No ano de 2015 participaram da pesquisa 198 estudantes, $54,4 \%$ do gênero feminino e $45,5 \%$ do gênero masculino. No ano de 2016, 148 estudantes participaram da pesquisa, também com predomínio de estudantes do gênero feminino $(52,7 \%)$. A faixa etária dos dois grupos foi semelhante, ambos com média de idade de 18 anos.

Um dado relevante e que difere significativamente entre os dois grupos é a quantidade de horas aula presenciais. Para os estudantes que cursaram a disciplina em 2015 foram ministradas 80 horas aula presenciais enquanto que em 2016 foram ofertadas 24 horas aula presenciais. Em 2015 foram realizadas 789 avaliações, aproximadamente 197 avaliações bimestrais, contra 587 avalições em 2016 e cuja média de avaliações por bimestre foi de 146.

O desempenho dos estudantes nos dois grupos estudados está descrito na tabela 3. Pode-se observar que as notas bimestrais dos estudantes na modalidade EC eleva-se gradualmente ao longo dos bimestres mas sempre são inferiores às notas dos estudantes na modalidade BL. De fato, os resultados dos estudantes do grupo $\mathrm{BL}$ foram significativamente melhores $(\mathrm{p}<0,05)$, incluindo a média final anual, excetuando-se o $4^{\circ}$ bimestre.

\section{DISCUSSÃO}

Nesta pesquisa foram avaliadas duas propostas de ensino para a disciplina de Bioquímica, uma delas tradicional, com 80 horas aula teóricas presenciais e outra na modalidade Blended Learning com 24 horas aula presenciais e com mistura de metodologias ativas $\mathrm{e}$ atividades on-line. Os resultados obtidos revelam que, a despeito do número menor de aulas presenciais, o curso Blended Learning apresentou melhores resultados.

As mudanças tecnológicas experimentadas nos últimos anos atingiram em cheio vários segmentos da sociedade moderna, incluindo a área de educação. A utilização de novas tecnologias associada às metodologias ativas de ensino mudou a perspectiva tanto de estudantes quanto de professores a respeito do conceito de sala aula pois permitiram a colaboração, comunicação e a interação de estudantes e professores fora daquele ambiente ${ }^{4}$. Hoje é inviável tentar ensinar sem utilizar esses recursos dentro da sala de aula, no entanto muitos desafios se abrem para que professores e estudantes se adaptem aos novos métodos.

Neste estudo foram propostas atividades online, tais como fórum de discussão, vídeo-aulas, e resolução de questões que deveriam ser realizadas antes das aulas presenciais. Essas atividades prévias foram acompanhadas por um professor tutor que estimulava e acompanhava a resolução das mesmas, além de ser responsável direto pela elucidação das dúvidas dos estudantes. No encontro presencial foram utilizadas somente metodologias ativas de aprendizagem: instrução por pares, atividades ativas, grupos de exercícios colaborativos e estudos de casos. 




Figura 1. Percurso de aprendizagem utilizado pelo estudante na modalidade Blended Learning

Tabela 1. Distribuição das atividades durante o bimestre. A cada bimestre foram estudados 2 capítulos do $e$-book, totalizando 8 capítulos em quatro bimestres

\begin{tabular}{cclccc}
\hline Aula & Nota & Atividade & $\begin{array}{c}\text { Valor da } \\
\text { Atividade }\end{array}$ & Atividades Propostas & $\begin{array}{c}\text { Capítulos } \\
\text { Estudados }\end{array}$ \\
\hline 1 & N1 & Aula Online & 1,0 & 01 questão do fórum de discussão & 1 \\
- & N2 & Aula Presencial & 1,5 & 01 atividade, metodologia ativa & 1 \\
2 & N3 & Aula Online & 0,5 & 05 questões objetivas & 1 e 2 \\
- & N4 & Aula Presencial & 1,5 & 01 atividade, metodologia ativa & 2 \\
3 & N5 & Aula Online & 0,5 & 05 questões objetivas & 2 \\
- & - & Aula Presencial & - & 01 atividade, metodologia ativa & 1 e 2 \\
4 & - & Aula Online & - & Quiz com 10 questões objetivas & 1 e 2 \\
5 & N6 & $\begin{array}{l}\text { Avaliação } \\
\text { Presencial }\end{array}$ & 5,0 & Avaliação presencial (presencial) & 1 e 2 \\
\end{tabular}

Tabela 2. Características dos dois grupos de estudantes analisados

\begin{tabular}{lcc}
\hline & 2015 & 2016 \\
& Grupo EC & Grupo BL \\
\hline Total de aulas presenciais & 80 & 24 \\
Número de estudantes & 198 & 148 \\
Número de avaliações & 789 & 587 \\
Idade Média dos Estudantes & $18 \pm 2,3$ anos & $18,3 \pm 2,5$ anos \\
Gênero & Feminino 108 & Feminino 78 \\
& Masculino 90 & Masculino 70 \\
\hline
\end{tabular}


Tabela 3. Distribuição das notas por bimestre

\begin{tabular}{|c|c|c|c|c|c|c|}
\hline Grupo & Ano & $\begin{array}{l}1^{0} \text { bimestre } \\
\text { Média } \pm \text { DP }\end{array}$ & $\begin{array}{l}2^{\circ} \text { bimestre } \\
\text { Média } \pm \text { DP }\end{array}$ & $\begin{array}{l}3^{0} \text { bimestre } \\
\text { Média } \pm \text { DP }\end{array}$ & $\begin{array}{l}4^{\circ} \text { bimestre } \\
\text { Média } \pm \text { DP }\end{array}$ & $\begin{array}{l}\text { Média anual } \\
\text { Média } \pm \text { DP }\end{array}$ \\
\hline $\mathrm{EC}$ & 2015 & $1,97 \pm 0,72^{\mathrm{c}}$ & $2,00 \pm 1,00^{c}$ & $2,12 \pm 0,90^{c}$ & $2,45 \pm 0,90^{\mathrm{a}, \mathrm{b}}$ & $2,12 \pm 0,90^{\mathrm{B}}$ \\
\hline BL & 2016 & $2,73 \pm 1,03^{\mathrm{a}}$ & $2,26 \pm 0,92^{\mathrm{b}, \mathrm{c}}$ & $2,64 \pm 1,24^{\mathrm{a}}$ & $2,26 \pm 0,93^{\mathrm{b}, \mathrm{c}}$ & $2,48 \pm 1,06^{\mathrm{A}}$ \\
\hline
\end{tabular}

A nota máxima em cada exame foi 5,0 pontos. As comparações entre os dois grupos de estudantes foram realizadas por meio do teste ANOVA. Diferenças entre letras indicam diferença estatística. Letras minúsculas indicam a comparação das médias bimestrais e letras maiúsculas a comparação entre a média anual.

Em todos os momentos, com exceção do $4^{\circ}$ bimestre, os estudantes do grupo BL obtiveram melhores resultados do que os estudantes do grupo EC, incluindo a média final que foi estatisticamente diferente entre os grupos. Sem dúvida, no ano de 2015, o grupo EC recebeu uma carga de conteúdo grande condensado em aulas presenciais, fato este que não ocorreu em 2016 visto que a utilização de diferentes metodologias possibilitou entregar informações de maneira gradual sem sobrecarga em um ou outro momento. Uma hipótese para explicar o pior desempenho dos estudantes do grupo EC é o de que a entrega de grandes quantidades de conteúdo durante uma única aula presencial poderia estar além da capacidade de memória de trabalho dos estudantes, criando uma carga cognitiva que não seria aproveitada adequadamente. Van Merriënboer e Sweller ${ }^{13}$ já utilizaram este argumento.

Um dado interessante observado foi a elevação gradual das notas do grupo EC ao longo do ano e que pode ser explicado pela pressão sofrida pelos mesmos com a proximidade do final do ano letivo. Esse aumento das notas, resultado aparentemente bom, revela um aumento de ansiedade e estresse por parte dos estudantes uma vez que, efetivamente precisavam de melhores notas para serem aprovados. Esse direcionamento pontual de recursos cognitivos para sair-se bem em avaliações diminui a capacidade mental dos estudantes para construir mais conhecimentos ${ }^{14} \mathrm{e}$ expõe uma deficiência nos métodos tradicionais de avaliação: a supervalorização da nota da prova em detrimento do ganho de aprendizagem.

Aqui cabe uma pergunta: por que a nota dos estudantes do grupo BL diminuiu no $4^{\circ}$ bimestre? Os feedbacks obtidos dos estudantes, tanto on-line quanto presencial, permitem sugerir que a preocupação com avaliações e entregas de atividades em outras disciplinas e a aquisição suficiente de nota na disciplina de Bioquímica, tenham sido responsáveis pela diminuição da média do $4^{\circ}$ bimestre. Também houve a percepção de que a distribuição dos conteúdos e as metodologias adotadas permitiram uma melhor formação dos estudantes. Isso foi percebido pela integração de conceitos adquiridos previamente com práticas subsequentes. Em outras palavras, as atividades passaram a ser formativas e não avaliativas. Minder et al. ${ }^{15}$ revelaram que as avaliações formativas vão além do ganho de conhecimento pontual. Elas permitem que o estudante correlacione $o$ conhecimento adquirido com disciplinas que ainda irá cursar.

Talvez o resultado mais importante observado nos dois anos de pesquisa não tenha sido convenientemente mensurado: o desenvolvimento de habilidades cognitivas, a colaboração entre os estudantes e a proatividade dos mesmos frente aos problemas propostos. Esses quesitos foram observados no grupo BL e corroboram com os 
resultados encontrados por outros pesquisadores que também implementaram a metodologia Blended Learning em disciplinas do curso de Odontologia ${ }^{16-}$ 17. Segundo esses mesmos autores, houve uma diferença significativa na efetividade das disciplinas em que a metodologia Blended foi implementada. De fato, a metodologia BL cria um ambiente de ensino que motiva o estudante ${ }^{4,18}$. Por outro lado, o professor deixa de ser apenas um transmissor de conteúdo, passando a ser um facilitador da aprendizagem.

O grau de satisfação dos estudantes não foi mensurado nesta pesquisa, mas, apesar da redução substancial no número de aulas presenciais, o que poderia causar um certo desconforto, os resultados mostram que houve um ganho de conhecimento. Obviamente existem barreiras que precisam ser transpostas para implementar definitivamente o método. Entre elas estão a criação de infraestrutura com salas apropriadas e a capacitação de professores o que demanda investimento financeiro por parte das instituições ${ }^{19,20}$, cultura organizacional e mudança de paradigmas por parte dos docentes ${ }^{2}$.

Finalmente, este estudo explorou o aproveitamento dos estudantes frente à utilização de novas metodologias de aprendizagem, não se preocupando em mensurar a satisfação dos estudantes. Nesse sentido, novas pesquisas devem ser conduzidas, mas é necessário que tanto estudantes quanto docentes percebam que a sala de aula física, como existe hoje, deixará de existir. A acessibilidade à informação permite que o estudante aprenda fora da sala de aula. Sendo assim, o momento dentro da sala de aula deve ser aproveitado para a formação dos estudantes. Para que isso ocorra, os docentes precisam ter interesse em implementar novas tecnologias nas suas disciplinas.

\section{CONCLUSÕES}

Os estudantes que cursaram a disciplina de Bioquímica no formato Blended Learning obtiveram melhores resultados do que os estudantes que utilizaram o modelo de ensino centrado em aulas presenciais.

\section{ABSTRACT \\ Implementation and assessment of a blended learning course in Biochemistry for Dental students}

The aim of the present study was to assess the performance of dental students in a blended learning course in biochemistry. Two groups were assessed: one made up of students who took the conventional course (CC) in biochemistry in 2015 , and one that attended the blended learning (BL) course in biochemistry in 2016. The learning goals, the syllabus, and the assessments were the same for both groups. Students' performance was checked by individual bimonthly classroom-based tests with similar levels of difficulty between the groups. The results were analyzed by two-way ANOVA and the level of significance was set at 0.05 . A total of 198 students (54.4\% female and $45.5 \%$ male) participated in the study in 2015, whereas 148 students $(52.7 \%$ female) participated in the study in 2016. Students from the BL group had a better performance in the 1st, 2nd, and 3rd bimesters $(p<0.05)$. The annual average of students in the BL group was also better than that of the CC group. This study therefore suggests that the blended learning method provides students with larger knowledge gain than does the classroombased method.

Descriptors: Education. Dentistry. Active Methodologies.

\section{REFERÊNCIAS}

1. Brasil. Conselho Nacional de Educação. Câmara de Educação Superior. Resolução CNES/CES 3, de 19 de fevereiro de 2002. Institui Diretrizes Curriculares Nacionais do Curso de Graduação em Odontologia. Diário Oficial da União: Brasília; 2002.

2. Tsai CC, Chai CS. The "third"-order barrier for technology-integration instruction: implications for teacher education. Australas J Educ Tec. 2012; 28(6):1057-60.

3. Ertemer AP. Addressing first- and second- 
order barriers to change: Strategies for technology integration. Educ Technol Res Dev. 1999; 47(4):47-61.

4. Fregona C, Sadza A. Blended learning. In: Enhancing Teaching Practice in Higher Education. 2016; 91-106.

5. Duhaney DC. Blended learning in education, training, and development. Performance Improvement. 2004; 43(8):35-8.

6. Bridges S, Chang JWW, Chu CH, Gardner K. Blended learning in situated contexts: 3-year evaluation of an online peer review project. Eur J Dent Educ. 2014; 18(3): 170-9.

7. Driscoll Blended learning: Let's get beyond the hype. E-learning. 2002; 1(4): 1-3.

8. Khalil MK, Abdel Meguid EM, Elkhider IA. Teaching of anatomical sciences: A blended learning approach. Clin Anat. 2018; 31(3):323-9.

9. Shang F, Liu CY. Blended learning in medical physiology improves nursing students' study efficiency. Adv Physiol Educ. 2018; 42(4):711-7.

10. Lang FK, Bodner GM. What the biochemistry education research literature can tell universitylevel biochemistry instructors. FASEB J. 2017; 31(1), Supplement 1.

11. Nelson DL, Cox MM. Princípios de Bioquímica de Lenhinger, 6a ed, 2014; Porto Alegre, Artmed.

12. Bloom, B. S.; Engelhart, M. D.; Furst, E. J.; Hill, W. H.; Krathwohl, D. R. (1956). Taxonomy of educational objectives: The classification of educational goals. Handbook I: Cognitive domain. New York: David McKay Company.

13. Van Merriënboer JJ, Sweller J. Cognitive load theory in health professional education: design principles and strategies. Med Educ. 2010; 44(1): 85-93.
14. Minder SP, Schmitz FM, Schär SG. Online formative assessment in a medical PBLcurriculum. In: Isaias P, Ifenthaler D, Sampson GD, Spector MJ, editors. Towards learning and instruction in Web 30: Advances in Cognitive and Educational Psychology. New York, NY: Springer New York; 2012

15. Minder SP, Weibel D, Wissmath B, Schmitz FM. Do students achieve the desired learning goals using open-book formative assessments? Int J Med Educ. 2018; 9:293-301

16. Pahinis K, Stokes CW, Walsh TF, Cannavina G. Evaluating a blended-learning course taught to different groups of learners in a dental school. J Dent Educ. 2007; 71(2): 269-78.

17. Maresca C, Barrero C, Duggan D, et al. Utilization of blended learning to teach preclinical endodontics. J Dent Educ. 2014; 78(8): 1194-204.

18. Rizvi NF, Gulzar S, Nicholas W, Nkoroi B. Barriers in adopting blended learning in a private university of Pakistan and East Africa: faculty members' perspective. M Health. 2017; (5):3-18.

19. Snoeyink R, Ertmer PA. Thrust into technology: how veteran teachers respond. J Educ Tech Syst. 2001; 30(1): 85-111.

20. Butler DL, Sellbom M. Barriers to adopting technology for teaching and learning. Educause Quarterly. 2002; 25(2): 22-8.

\section{Correspondência para:}

João Armando Brancher

e-mail: brancher.a@gmail.com

Universidade Positivo

Rua Prof. Pedro Viriato Parigot de Souza, 5300

81280-330 Curitiba/PR 